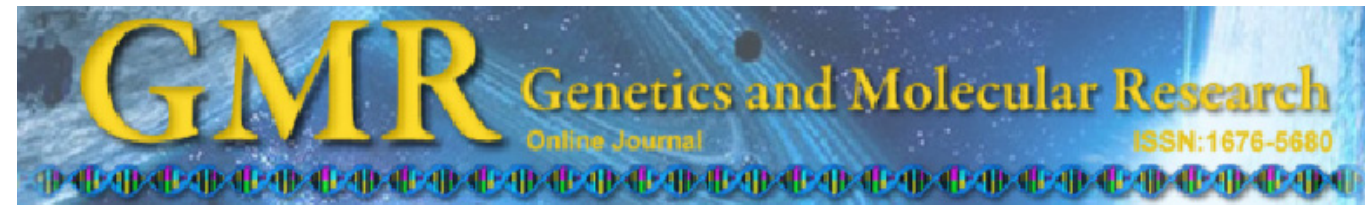

\title{
Isolation of fertility-related genes of multiple- allele-inherited male sterility in Brassica rapa ssp pekinensis by cDNA-AFLP
}

\author{
R.Q. Ji, Q. Song, X.F. Xin, X. Zhou and H. Feng \\ Department of Horticulture, Shenyang Agricultural University, \\ Shenyang, China \\ Corresponding authors: H. Feng / R.Q. Ji \\ E-mail: fenghuiaaa@263.net / ruiqinji@yahoo.com.cn
}

Genet. Mol. Res. 10 (4): 4073-4083 (2011)

Received June 16, 2011

Accepted October 19, 2011

Published December 5, 2011

DOI http://dx.doi.org/10.4238/2011.December.5.8

\begin{abstract}
To better understand the molecular mechanisms of multiple-allele-inherited male sterility in Chinese cabbage (Brassica rapa ssp pekinensis), differentially expressed genes in fertile and sterile plants must be isolated. We used cDNA-AFLP analysis to isolate differentially expressed genes in fertile and sterile buds of the twotype line, AB01. Sixteen high-quality sequences were generated, 11 of which were up-regulated in fertile buds, and five of which were upregulated in sterile buds. Based on BLAST screening and functional annotation, these genes have homology with genes encoding known flower- or bud-specific proteins, metabolism-related proteins and cellstructure proteins. In addition, the full-length cDNA sequences of the actin gene were cloned from the cabbage plants by RACE and used as an internal standard for semi-quantitative reverse transcription-PCR. Expression of three flower- or bud-specific differentially expressed transcript-derived fragments in fertile and sterile buds was examined using RT-PCR; the expression patterns of these genes were similar to the patterns observed in the cDNA-AFLP analysis.
\end{abstract}

Key words: Chinese cabbage; Male sterility; Fertility-related genes; cDNA-AFLP 


\section{INTRODUCTION}

Chinese cabbage (Brassica rapa L. ssp pekinensis) is a typical allogamous plant with bisexual flowers and obvious heterosis. The utilization of male sterile lines is an economical and stable method of improving cross-breeding in Chinese cabbage. Feng et al. (1995) obtained four genetically stable lines of Chinese cabbage comprising $100 \%$ male sterile plants by crossing male sterile plants with male fertile plants between $\mathrm{AB}$ lines. Then, a classical genetic analysis was designed and carried out to reveal the inheritance mechanism of the male sterility locus, and a genetic hypothesis of a genic multiple allele male sterile gene was proposed (Feng et al., 1996). This hypothesis was able to satisfactorily explain most of our data. This model proposes the existence of a single locus with three alleles: the " $M s$ " allele for male sterility, the " $m s$ " allele for fertility, and the " $M s^{f}$ " for fertility restoration. The dominant-recessive relationship among these alleles was proposed to be $M s^{f}>M s>m s$. According to this genetic model, in order to obtain a male sterile line with $100 \%$ male sterile plants (Msms), a temporary maintainer line (msms) should be crossed with the homozygous sterile line (MsMs) (Figure 1). Hybrid seed production could be subsequently carried out by using this population as the female parent. Thus, the manual removal of fertile plants in the sterile line, a necessary step in a two-line system, could be obviated. Following this model, the male sterile allele could easily be transferred into normal fertile lines. Thus, this allele could be used to breed new male sterile lines in Chinese cabbage. This model may also be used to guide research on male sterility in other crops, such as oilseed rape (Song et al., 2006).

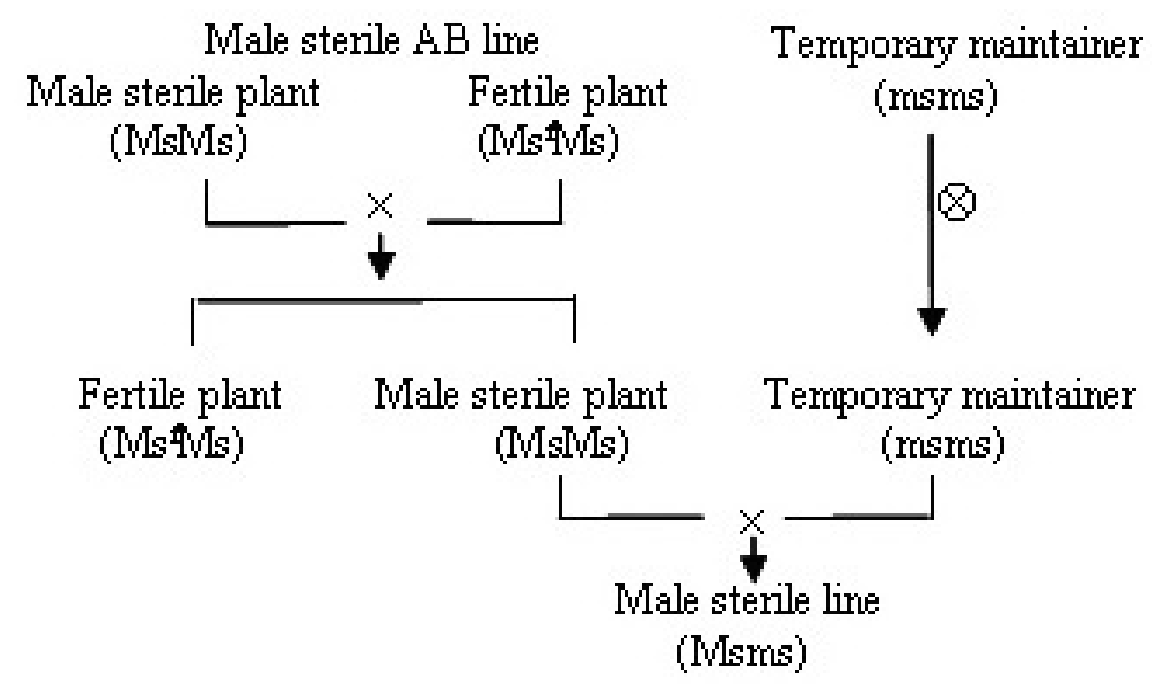

Figure 1. Genetic model of multiple allele male sterile line of Chinese cabbage.

The model proposed by Feng et al. (1996) has been shown to be practicable for hybrid seed production. The allele has been transferred to Chinese cabbage of different ecotypes (Wang et al., 2005, Feng et al., 2007). Some high quality male sterile lines have been bred successfully. With these male sterile materials, two commercial hybrids have been registered and released in China, and some molecular markers have subsequently been identified (Feng 
et al., 2009; Wei et al., 2009; Wang et al., 2010). To make better use of these high-quality male sterile lines, it is crucial to isolate fertility-related genes to help understand the genetic mechanism of male sterility.

cDNA-amplified fragment length polymorphism (cDNA-AFLP) is a common technique used to isolate highly abundant differentially expressed genes and to amplify low-abundance gene signals. cDNA-AFLP has been widely used to isolate differentially expressed genes in sterile and fertile tissue from several plants, such as genic sterile gourds (Subhash et al., 2008). Preferentially expressed genes in pollen were identified by differential display reverse transcription-polymerase chain reaction (DDRT-PCR) and cDNA-AFLP (Cao et al., 2006; Liu et al., 2006; Zhang et al., 2008). The fertility-related genes associated with genic multiple-allele-inherited male sterility in Chinese cabbage have not been identified, which is necessary in order to determine the molecular mechanisms that underlie multiple-allele-gene controlled male sterility.

In this study, highly abundant differentially expressed genes between fertile and sterile Chinese cabbage plants of the A/B line AB01 were obtained using cDNA-AFLP technology. Reverse transcription polymerase chain reaction (RT-PCR) initially developed by Tu et al. (1997) is an effective technique to quantify the ratio of mRNA and has been used to study mRNA regulation (Kim et al., 1998). In this study, three differentially expressed transcriptderived fragments (TDFs) identified in fertile and sterile buds were verified using RT-PCR.

This study provides a basis for the further investigation on the transcriptome of multiple-allele-gene controlled male sterility in the Chinese cabbage.

\section{MATERIAL AND METHODS}

\section{Plant materials}

$\mathrm{AB} 01$, a previously bred $\mathrm{AB}$ line of Chinese cabbage (Brassica rapa L. ssp pekinensis) was used as the study material. The GMS A/B 'AB01' line generates a 1:1 proportion of fertile (AB01-2, $\left.M s^{f} M s\right)$ and sterile (AB01-1, MsMs) progeny and is therefore considered to be a stable system. In this study, the GMS A/B 'AB01' line was reproduced continuously by a sister-line cross $(\mathrm{A} \times \mathrm{B}, \mathrm{nA} \times \mathrm{B})$ for more than 10 years. The seeds were sown in the field station of Shenyang Agriculture University, China. During the flowering stage, flower buds were divided into different grades according to filament length, anther color, and presence or absence of pollen, and then harvested quickly on ice. All harvested samples were snap-frozen in liquid nitrogen and stored at $-5^{\circ} \mathrm{C}$ prior to use.

\section{Total RNA extraction and mRNA isolation}

Total RNA was extracted from the buds of six fertile or six sterile plants using the RNAprep pure Plant Kit (TianGen, Beijing, China) according to the manufacturer protocol; each extraction was repeated three times. Total RNA was dissolved in diethyl pyrocarbonate (DEPC)-treated water and stored at $-85^{\circ} \mathrm{C}$. The yield and quality of total RNA were determined using a DU 800 UV/Visible Spectrophotometer (Beckman, Fullerton, CA, USA) at 260 and $280 \mathrm{~nm}$. The integrity and quality of total RNA and mRNA were verified by denaturing agarose gel electrophoresis on 1.0\% agarose ethidium bromide stained gels and imaged using GeneSnap (SynGene, Cambridge, UK). 


\section{cDNA-AFLP analysis}

Double-stranded cDNAs were synthesized from fertile and sterile mRNA using the M-MLV reverse transcriptase kit (TakaRa, Shiga, Japan) following the manufacturer protocol. Double-stranded cDNA was digested using the EcoRI and MseI restriction enzymes. AFLP reactions were performed according to the method of Wei et al. (2009) using a total of 256 primer combinations derived from $16 E c o R I+\mathrm{NN}$ and $16 \mathrm{MseI}+\mathrm{NNN}$ primers. All amplifications were performed in an iCycler (Bio-Rad, Hercules, CA, USA) using the PCR conditions described by Vos et al. (1995). PCR products were mixed with an equal volume of loading dye, denatured at $94^{\circ} \mathrm{C}$ for $5 \mathrm{~min}$ and separated on a $6 \%$ denaturing polyacrylamide gel. After electrophoresis, the gel was developed using a silver staining kit (Bioneer, Daejeon, Korea). Gel pieces containing differentially expressed transcript-derived fragments (TDFs) were sliced and boiled for $5 \mathrm{~min}$ in $100 \mu \mathrm{L}$ sterile water. The eluted cDNA fragments were re-amplified using standard PCR methods, electrophoretically separated on agarose gels, purified using a gel extraction kit (Tiangen, Beijing, China), and cloned into the pGEM-T Easy Vector system I (Promega, Fitchburg, WI, USA). The cDNA-AFLP clones were sequenced by Shanghai Sangon Biological Engineering Technology \& Services, Co., Shanghai, China.

\section{BLAST analysis}

The vector and adaptor sequences were removed from the differentially expressed cDNA-AFLP sequences to obtain TDFs. TDFs were compared with translated nucleotide databases using BLASTN and BLASTX (http://blast.ncbi.nlm.nih.gov).

\section{The achievement of actin gene fragments}

Primers for the actin gene (forward: 5'-ATCTACGAGGGTTATGCT-3'; reverse: 5'-CCACTGAGGACGATGTTT-3') were designed according to the homologous sequence of Brassica napus actin gene (GQ339782.1) and Arabidopsis thaliana ACT7(NM_121018.3); fragments were synthesized by Shanghai Sangon Biological Engineering Technology \& Services, Co., Shanghai, China. First-Strand cDNA was synthesized with SuperScript ${ }^{\mathbb{B}}$ III reverse transcriptase (Invitrogen, USA) from the total RNAs of fertile and sterile Chinese cabbage buds. PCR were performed in a total volume of $25 \mu \mathrm{L}$, which contained $170 \mathrm{ng}$ cDNA, 1.25 mmol dNTPs, $10 \mu \mathrm{mol}$ each primer, $37.5 \mathrm{mmol} \mathrm{MgCl}_{2}$, and $0.2 \mathrm{U} \mathrm{Taq}$ DNA polymerase (Henan Sino-American Biotechnology Co. Ltd., China). The PCR cycling parameters included an initial $94^{\circ} \mathrm{C}$ for $5 \mathrm{~min}, 27$ cycles of $94^{\circ} \mathrm{C}$ for $1 \mathrm{~min}, 54^{\circ} \mathrm{C}$ annealing for $30 \mathrm{~s}$ and $72^{\circ} \mathrm{C}$ for $50 \mathrm{~s}$, and a final extension of $72^{\circ} \mathrm{C}$ for $10 \mathrm{~min}$. Fragments amplified from the RTPCR were cloned into pGEM-T Easy vectors (Promega, USA) and sequenced. Gene-specific primers for the 5'- and 3'-RACE rapid amplification of cDNA ends (RACE) reactions were designed according to the sequences of the fragments. RACE reactions were performed using a SMARTer ${ }^{\mathrm{TM}}$ RACE cDNA Amplification Kit following the manufacturer protocol (Clontech, Santa Clara, CA, USA).

\section{Analysis of gene expression by semi-quantitative RT-PCR}

Three flower- or bud-specific genes were used to detect the validity of cDNA-AFLP. 
The experiment was repeated three times. Each time, fertile RNAs of six fertile plants or sterile RNAs of six sterile plants were pooled respectively and regulated to be consistently identical using the DU800. Bulked RNAs were reverse-transcribed into first 1st-strand cDNA and the one-step RT-PCR protocol was performed using M-MLV reverse transcriptase according to manufacturer instructions (Promega), the consistence of first strand cDNA was regulated to identical before PCR. The actin gene was used as a reference. TDF primers for RT-PCR analysis were generated following the cDNA-AFLP sequencing results. PCR reactions and progress were performed as previously mentioned, but the annealing temperature was adjusted according to different primers. The primer sequences, annealing temperature and product length used for RT-PCR are listed in Table 1.

Table 1. The primer sequences, product length and annealing temperatures for RT-PCR analysis.

\begin{tabular}{|c|c|c|c|}
\hline Gene & Primer sequence $\left(5^{\prime}-3^{\prime}\right)$ & Amplicon (bp) & Annealing $\mathrm{T}\left({ }^{\circ} \mathrm{C}\right)$ \\
\hline \multirow[t]{2}{*}{$F C l$} & GGGTGTTGCCATAGGAGTTG & 136 & 56 \\
\hline & GGTTGAAGCCGTGAAGTTTG & & \\
\hline \multirow[t]{2}{*}{$F C 2$} & TTACGTTCAACCCTGCTT & 213 & 54 \\
\hline & CGATACCACCTAATTTACACT & & \\
\hline \multirow[t]{2}{*}{$F C 3$} & ACCAAACAATGGGAGTGC & 178 & 54 \\
\hline & GCTGTGACATGCTGCTT & & \\
\hline \multirow[t]{2}{*}{$\beta$-actin } & ATCTACGAGGGTTATGCT & 412 & 54 \\
\hline & CCACTGAGGACGATGTTT & & \\
\hline
\end{tabular}

\section{RESULTS}

\section{Quality and purity of total RNA}

Electrophoresis gel imaging revealed that total RNA typically showed two bright bands (Figure 2), which corresponded to ribosomal 28S and 18S RNA with A260/A280 values that ranged from approximately 1.82 to 1.95 , indicating that the RNA purity was sufficiently high.

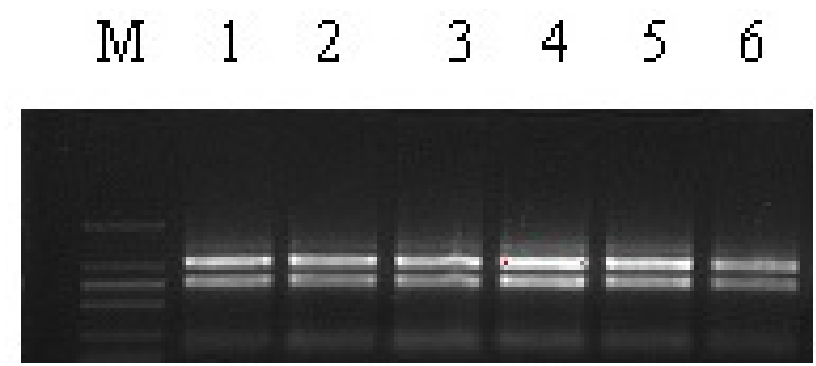

Figure 2. Total RNA electrophoresis of fertile and sterile buds representing stages. Lane $M=$ molecular marker; lanes 1-3 = fertile buds; lanes 4- $6=$ sterile buds.

\section{Differentially expressed TDFs obtained by cDNA-AFLP}

Silver-stained cDNA-AFLP analysis was performed to compare differences in gene expression between fertile and sterile cDNA pools from the A/B line AB01 plants. Approximately $35-45$ bands between 1000 and $100 \mathrm{bp}$ were observed with each combination of primers (Figure 3). Six-hundred TDFs of fertile and sterile buds (Brassica rapa L. ssp pekinensis) were 
obtained using EcoRI $+2 / M s e I+3$ primer combinations for selective amplification. A total number of 175 (29\%) TDFs were differentially expressed. These TDFs were excised from the gels, and 16 were successfully re-amplified, cloned and sequenced.

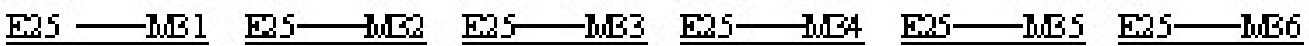

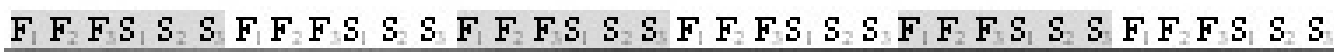

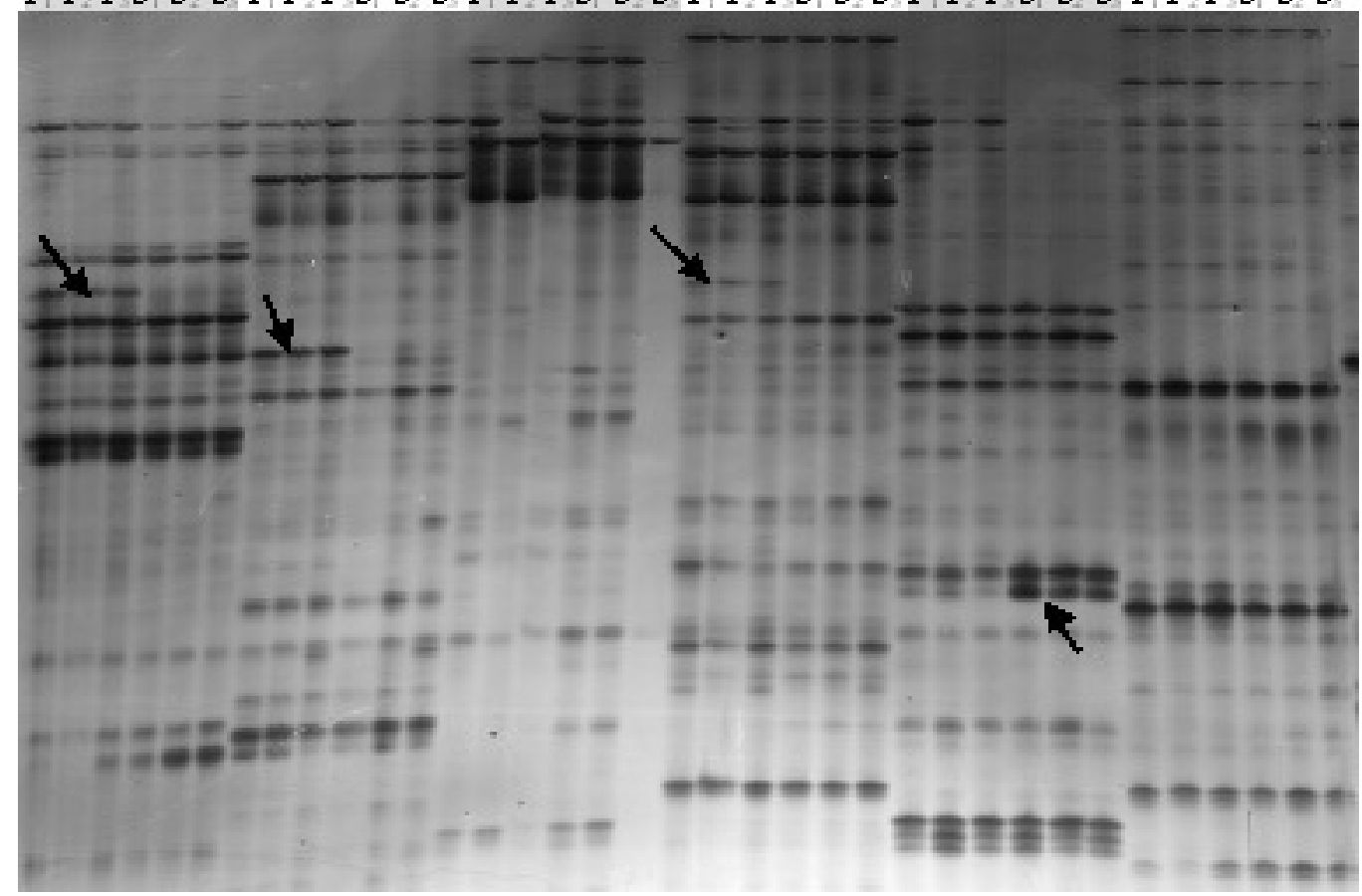

Figure 3. A representative photograph of a silver-stained cDNA-AFLP gel showing the differential expression of the genes between fertile and sterile buds. An arrow indicates the band that was excised from the gels.

\section{Functional analysis of differentially TDFs}

A total of 16 differentially expressed TDFs, ranging in size from 70 to $352 \mathrm{bp}$, were obtained after low-quality and repeated sequences were eliminated. Those 16 sequences were aligned individually against the Whole Genome Shotgun (WGS) database using the BLASTN tool; this showed that 11 TDFs were up-regulated in fertile buds and five TDFs were upregulated in sterile buds. All of the TDFs were homologous to known sequences deposited in the National Center for Biotechnology. Of these, 14 TDFs were homologous to expressed sequence tags from Arabidopsis thaliana or Brassica. The genes with homology to known sequences could be divided into several functional categories: three were related to flower- or bud-specific proteins; three were lipid metabolism proteins, of which one was energy metabolism protein and another was secondary metabolic-related protein; two cell structure protein; and two other proteins (one Expansin and one oleosin-like protein). Four were proteins of unknown function (Table 2). 
Fertility-related genes in Brassica rapa ssp pekinensis

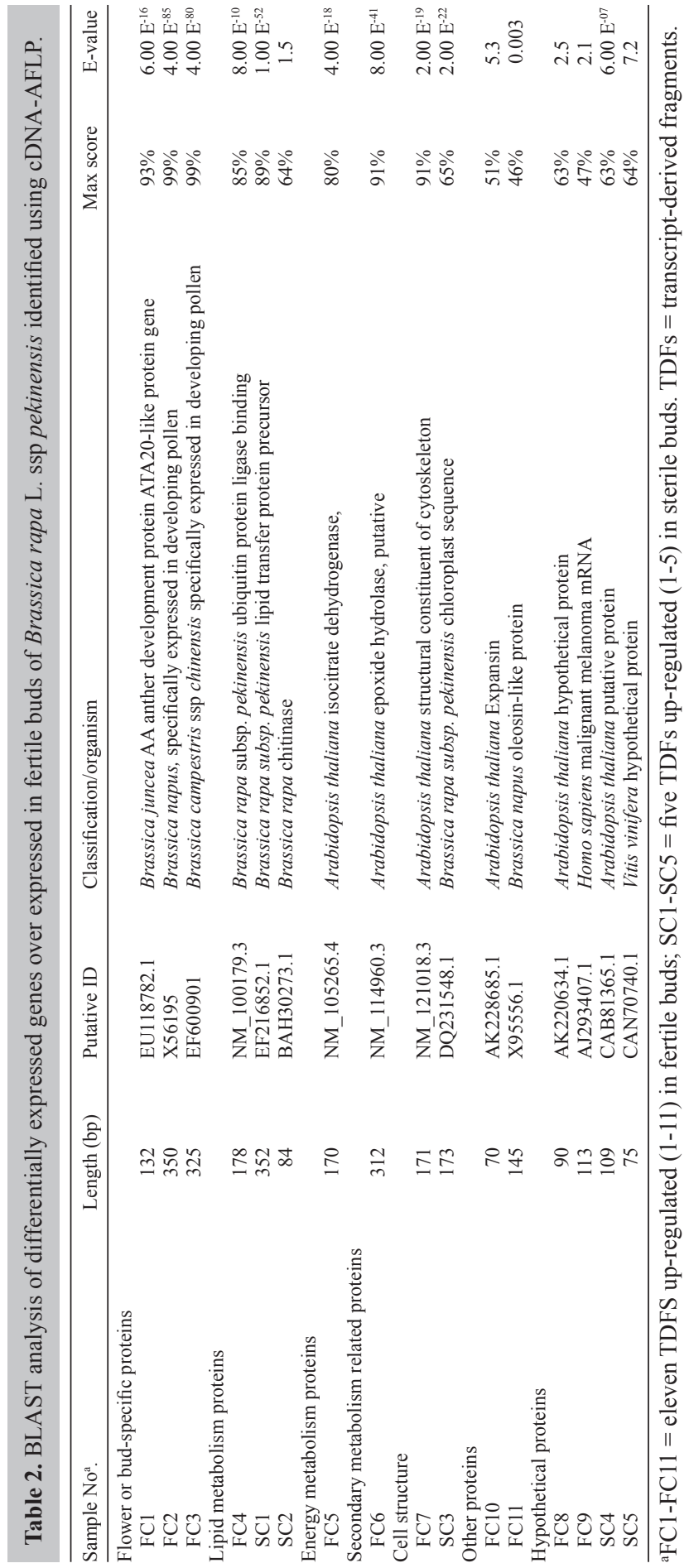




\section{Actin fragment sequences in Chinese cabbage}

RT-PCR amplified an approximately 402-bp cDNA fragment of the actin gene from Brassica rapa L. ssp pekinensis. Thereafter, 3-RACE and 5-RACE were performed on fertile and sterile samples and the full-length actin gene sequences were obtained with a length of 1465 bp. Database comparison results indicated that these sequences are the sequence of actin gene. The sequences of the actin gene in both the fertile and sterile plants were uniform and named as Brlact7 (GenBank ${ }^{\circledR}$ accession No. JN120480). The deduced 377 amino acid sequence showed a high identity to that of Arabidopsis thaliana actin-7 (Atactin7) and Brassica napus actin (Bnactin) (Figure 4).

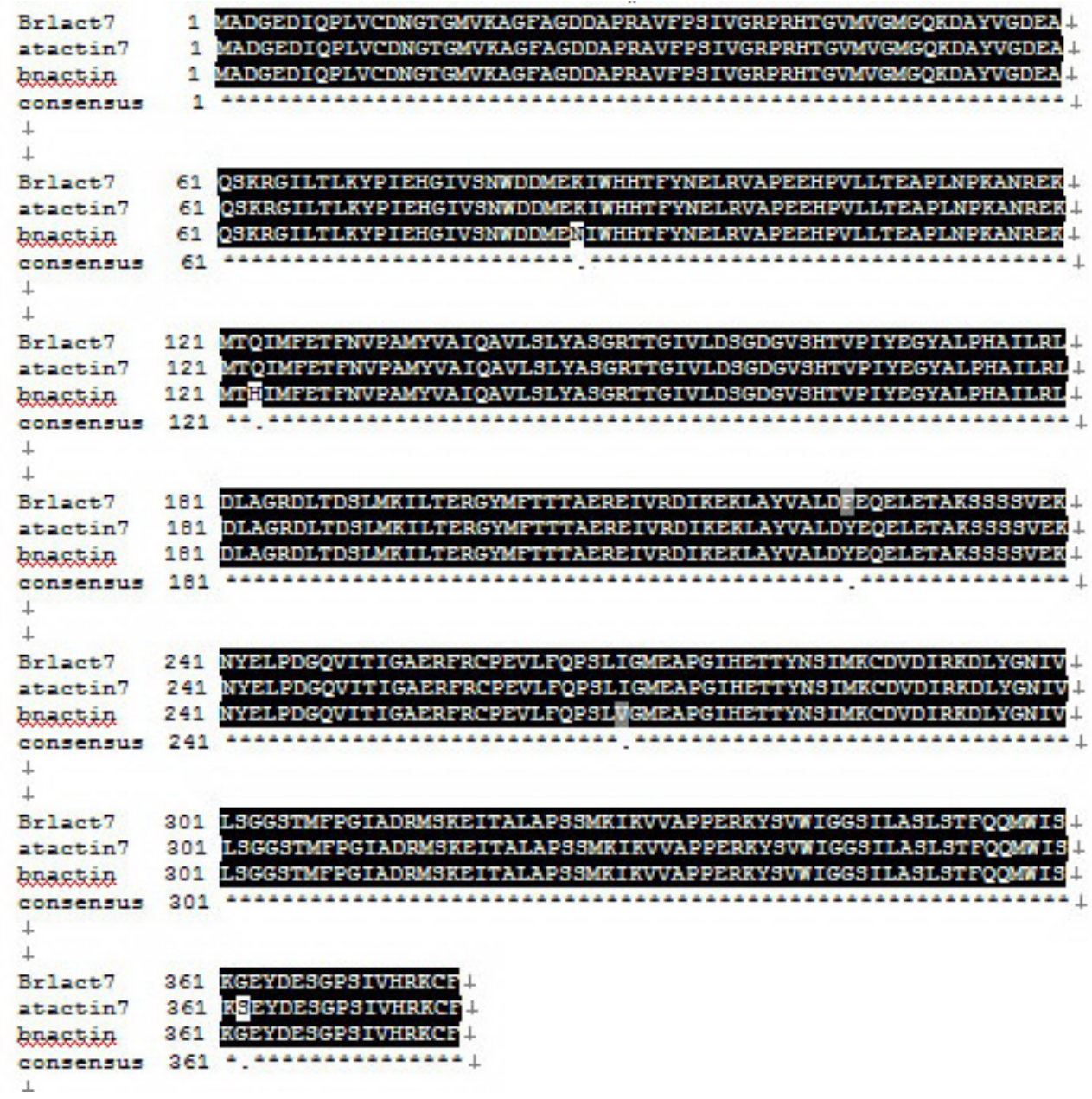

Figure 4. Amino acid alignment of predicted actin proteins of Brassica rapa L. ssp pekinensis, Arabidopsis thaliana and Brassica napus. Sequences were aligned using ClustalX 11.83 without any manual adjustment. The similarity and dissimilarity sequences are shown as "*” and ".:", respectively. Brlact7, atactin7 and bnactin are the actin proteins of Brassica rapa L. ssp pekinensis, Arabidopsis thaliana and Brassica napus, respectively. 


\section{RT-PCR assay}

The actin genes had coincident expression between fertile and sterile buds, which were used as a housekeeping gene in this research. Three flower- or bud-specific TDFs were selected and their expression patterns were verified using RT-PCR. Three TDFs (FC1, FC2 and FC3) were expressed at higher levels in fertile buds (Figure 5). The expression patterns of these genes were similar to the patterns observed in the cDNA-AFLP results.

A

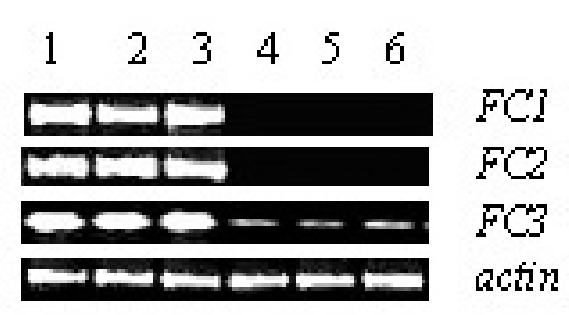

B

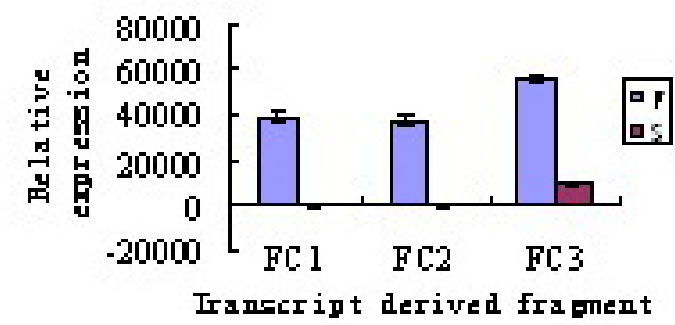

Figure 5. Confirmation of three flower or bud-specific flower or bud-specific protein TDFs that were identified using cDNA-AFLP expression patterns in fertile (F) and sterile buds (S) using RT-PCR. FC1 (136 bp), FC2 (213 bp), FC3 (178 bp) and the reference gene, actin. The experiments were executed three times, and six bulked buds were used in each time. A. Visualization of the transcript-derived fragment in the cDNA-AFLP gel autoradiogram (A) and validated by semi-quantitative RT-PCR analysis. 1-3, triplicate experiments in fertile buds; 4-6, triplicate experiments in the sterile buds. B. Semi-quantitative RT-PCR showing the expression levels for selected TDFs that are indicated below the $\mathrm{x}$-axis. Columns show different expression levels between fertile and sterile buds, and bars indicate standard deviations. The experiment was performed three times with identical RNA samples, obtaining similar results.

\section{DISCUSSION}

Fertile and sterile plants of the two-type Chinese cabbage line, AB01, have similar genetic backgrounds (Feng et al., 2009), which provides an ideal model to evaluate the molecular mechanisms that underlie genic multiple-allele-inherited male sterility. In this study, a total of 16 differentially expressed TDFs were found in fertile and sterile plants, which confirmed a high degree of genetic similarity between the two sets of materials. Wu et al. (2007) have reported that fertile and sterile plants have a similar gene expression pattern up to the key stage that precedes fertility control. When development of the male gametes stops in sterile buds, the number of differentially expressed genes increased; however, after the fertility control stage in normal fertile buds, large numbers of genes were expressed.

Abnormal flower or bud development is a common phenomenon in male sterile plants, such as B. rapa L.ssp pekinensis (Guo et al., 2001), Zinnia elegans (Ye et al., 2008), B. napus L. (Dong et al., 2004), and Capsicum annum L. (He et al., 2008). In this study, the sequences of three flower- or bud-specific genes were detected in the 16 TDF homologous sequences, which indicated that altered flower or bud development may be an important process in Chinese cabbage multiple-allele nuclear genic male sterility. 
Our study revealed that certain genes related to metabolism are differentially expressed in Chinese cabbages with multiple-allele nuclear genic male sterility. Of the 16 TDF homologous sequences identified in this study, five were metabolism genes of which three (F4, F5 and F6) were up-regulated in fertile buds and two (S1 and $S 2)$ were up-regulated in sterile buds (Table 2). Three of the six differentially expressed metabolism-related genes are related to lipid metabolism. Lipid metabolism is an important process in male gametogenesis, as shown by Shi (2007), who demonstrated that the differential regulation of the rice lipid metabolism-related gene, $O s M S 2$, can lead to sterility. In addition, a relationship has been suggested between sterility in Lycium fruit (Mi et al., 2008) and a type of physiological sterility in wheat caused by a shortage in the supply of cellular energy. Furthermore, studies have shown reduced levels may directly affect male sterility in maize (Xia and Liu, 1994) and cytoplasmic male sterility in beet (He and Tian, 2008). In this study, three lipid metabolism and one energy metabolism genes were identified as differently expressed (Table 2), which suggested that altered pathways of lipid and energy metabolism may be associated with genic multiple-allele-inherited male sterility in Chinese cabbage. However, these conclusions need to be verified by further research.

Additionally, two differentially expressed genes related to cell structure were identified. It is possible that the biogenesis of cellular components can affect genic multiple-alleleinherited male sterility in Chinese cabbage. However, hardly any related research exists, with the exception of the study by Schroder and Kaufman (2005), who indicated that protein folding is very important for normal development in budding yeast.

The utilization of genic multiple-allele inherited male sterility in cross-breeding programs is an economical and stable approach to breed new varieties of Chinese cabbage. It is important to understand the genetic expression patterns and regulatory mechanisms that control genic multiple-allele inherited male sterility in order to apply these concepts in practice. In this study, we deduced that genic multiple-allele-inherited male sterility in Chinese cabbage is associated with the altered expression levels of flower- or bud-specific genes. Metabolism-related genes were particularly affected. However, the full details of the complicated molecular mechanisms that govern male sterility remain elusive. Further studies are needed to isolate and identify the function of increasing numbers of genes involved in fertility to help understand the genetic mechanisms of male sterility.

\section{ACKNOWLEDGMENTS}

Research supported by grants from the National Natural Science Foundation of China (\#30800748), the China Postdoctoral Science Foundation (\#20070411080), the Liaoning Province Doctor Startup Foundation of China (\#20071059) and the Liaoning Province Education Administration Foundation of China (\#2009s090).

\section{REFERENCES}

Cao JS, Yu XL, Ye WZ, Lu G, et al. (2006). Functional analysis of a novel male fertility CYP86MF gene in Chinese cabbage (Brassica campestris L. ssp chinensis Makino). Plant Cell Rep. 24: 715-723.

Dong JG, Dong ZS, Liu XX and Liu CS (2004). Cytological studies on anther development of ecological male sterile line 533S in Brassica napus L. J. Northwest Sci. Tech. Univ. Agr. For. 32: 61-66.

Feng H, Wei YT and Zhang SN (1995). Inheritance of and utilization model for genic male sterility in Chinese cabbage 
(Brassica pekinensis Rupr.). Acta Hortic. 402: 133-140.

Feng H, Wei YT and Ji SJ (1996). Multiple allele model for genic male sterility in Chinese cabbage. Acta Hortic. 467: 133-142.

Feng H, Xu W and Wang YG (2007). Directive transfer of the genetic male sterile line of milk Chinese cabbage AI023. Acta Hortic. Sin. 34: 659-664.

Feng H, Wei P, Piao ZY, Liu ZY, et al. (2009). SSR and SCAR mapping of a multiple-allele male-sterile gene in Chinese cabbage (Brassica rapa L.). Theor. Appl. Genet. 119: 333-339.

Guo JX, Sun RF, Song JX and Zhang SJ (2001). Microsporogenesis of several male sterile lines in Brassica rapa L. ssp. pekinensis. Acta Hortic. Sin. 28: 409-414.

He CZ, Liu ZM, Xion XY and Zou X (2008). Cytologic observations tions on anther development of 9704A, a cytoplasmic male sterile line in Capsicum annum L. Acta Hortic. Sin. 35: 521-528.

Kim SW, Harney JW and Larsen PR (1998). Studies of the hormonal regulation of type 25 '-iodothyronine deiodinase messenger ribonucleic acid in pituitary tumor cells using semiquantitative reverse transcription-polymerase chain reaction. Endocrinology 139: 4895-4905.

Liu LC, Cao JS, Yu XL and Xiang X (2006). Expression of an antisense BcMF3 affects microsporogenesis and pollen tube growth in Arabidopsis. Agr. Sci. China 5: 339-345.

Mi HL, Zhang XY, Fan YF and Li YK (2008). A study on activated oxygen metabolism in the male sterile plants of Lycium barbarum. Acta Agr. Univ. Jiangxiensis 30: 796-798.

Schroder M and Kaufman RJ (2005). The mammalian unfolded protein response. Annu. Rev. Biochem. 74: 739-789.

Shi J (2007). Mapping of a Rice (Oryza sativa L.) Fatty Acyl Reductase OsMS2 Gene and its Function in Pollen wall Development. Master's thesis, Shanghai Jiaotong University, Shanghai.

Song LQ, Fu TD, Tu JX and Ma CZ (2006). Molecular validation of multiple allele inheritance for dominant genic male sterility gene in Brassica napus L. Theor. Appl. Genet. 113: 55-62.

Subhash KR, Gaurab G, Kaushik G and Sanjukta D (2008). A cDNA-AFLP approach to look for differentially expressed gene fragments in dioecious pointed gourd (Trichosanthes dioica Roxb.). Res. Commun. 94: 381-385.

Tu HM, Kim SW, Salvatore D, Bartha T, et al. (1997). Regional distribution of type 2 thyroxine deiodinase messenger ribonucleic acid in rat hypothalamus and pituitary and its regulation by thyroid hormone. Endocrinology 138: 33593368.

Vos P, Hogers R, Bleeker M, Reijans M, et al. (1995). AFLP: a new technique for DNA fingerprinting. Nucleic Acids Res. 23: 4407-4414.

Wang LL, Wei P, Liu ZY and Li CY (2010). SSR mapping of the Msf, a multiple-allele male-fertility restorer gene in Chinese cabbage (Brassica campestris L. ssp. Pekinensis). Acta Hortic. Sin. 37: 923-930.

Wang YG, Feng H, Lin GR and Xu SF (2005). The transfer of genetic male sterile lines in Brassica campestris L. ssp. chinesis (L.) Makino. Acta Hortic. Sin. 32: 628-631.

Wei P, Feng H, Piao ZY and Li CY (2009). Identification of SSR markers linked to a genic multiple-allele male sterile gene in Chinese cabbage. Acta Hortic. Sin. 36: 103-108.

Wu JY, Shen JR, Mao XZ and Liu KD (2007). Isolation and analysis of differentially expressed genes in dominant genic male sterility (DGMS) Brassica napus L. using subtractive PCR and cDNA microarray. Plant Sci. 172: 204-211.

Xia T and Liu JL (1994). Cytochrome oxidase activity and ATP content of male-sterile cytoplasm in maize (Zea mays L.). Acta Agr. Boreali-Sin. 9: 33-37.

Ye YM, Hu QS, Chen TH and Bao MZ (2008). Male sterile lines of Zinnia elegans and their cytological observations. Agr. Sci. China 7: 423-431.

Zhang Q, Cao J, Huang L, Xiang X, et al. (2008). BcMF5, a pollen coat protein gene (PCP), from Brassica rapa. ssp. chinensis, involved in the transcription of different lengths of 3'-UTRs of PCPs. Mol. Biol. Rep. 35: 439-445. 